\title{
University College London (UCL) Symposia on Neonatal Neurology and Neuro Protection
}

\author{
SUdHIN THAYYIL* AND NICOLA J ROBERTSON \\ Translational Neonatal Medicine, UCL Institute for Women's Health, 86-96 Chenies Mews, London WC1E 6HX, UK. \\ Correspondence to: Sudhin Thayyil, 15 Grafton Drive, Cambridge CB23 7UE,UK. s.thayyil@ucl.ac.uk
}

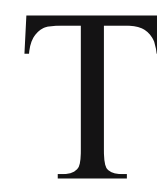

The last six decades have witnessed significant advances in the understanding and delivery of neonatal intensive care. Survival rates have improved dramatically and care now involves sicker and more immature babies. However, improvements in neurological monitoring techniques and in neurodevelopmental and cognitive outcome have not kept pace with improved survival.

Brain oriented neonatal intensive care requires a high degree of technical and clinical expertise, sophisticated neuroimaging and electrophysiological investigations. Such an approach is crucial for several reasons: $(a)$ the newborn brain is in a developing state and many of the currently used treatment modalities can cause gross or subtle brain injury; (b) unlike damage to other organ systems, brain injuries may not be apparent until several years later and are irreversible; and (c) long term outcome measurements are lacking in many neonatal units, particularly in developing countries.

During the history of neonatal medicine, there have been several instances of inadvertent iatrogenic harm: for example, oxygen therapy to reduce apnea in the 1940s led to retrolental fibroplasia; more recently, an increased incidence of cerebral palsy was observed in babies born to mothers treated with antibiotics for premature labor. By the nature of the speciality, neonatologists are always keen to embrace the latest treatment modalities available; unfortunately many of these come at a price, which may not be apparent unless rigorous scientific evaluation is undertaken.
Moreover, certain therapies that are safe and efficacious in one setting may be unsafe in another. One such therapy that has recently emerged from bench to bedside in developed countries is therapeutic hypothermia; however, extrapolation of the evidence that this is a safe and effective therapy in low resource and translational countries is problematic and clinicians should adhere to the principle of 'do no harm first'

As a part of UCL-India initiative, University College London conducted two symposia on neonatal neurological investigations and neuroprotection (www.isnnth.com) in India, in December 2009. Deliberations from these symposia will be published as a series of state-of-the-art review articles in Indian Pediatrics, over the next few months.

The topics covered will include ethical aspects of therapeutic hypothermia, cranial ultrasound, magnetic resonance imaging and spectroscopy, cerebral function monitoring, electroencephalography, optical imaging and near infrared spectroscopy. Many of these investigations are now available in India, or likely to be used in clinical practice in the near future.

Competing interest: None.

Funding: ST is funded by the Department of Health (UK) and Comprehensive Biomedical Research Centre (CBRC), University College London. NJR is funded by Higher Education Funding Council for England (HEFCE).

* This symposia is dedicated to the memory of my father, Dr TV Sudhan, who always wanted me to work for the babies in India. 